\title{
5 Psychologische Diagnostik und Therapie in der andrologischen Liaisonsprechstunde
}

\author{
Kurt Seikowski
}

Seit den sechziger Jahren des vorigen Jahrhunderts hat sich am Leipziger Universitätsklinikum eine Andrologische Sprechstunde etabliert, die bei Zeugungsfähigkeitsproblemen (Impotentia generandi) und sexuellen Störungen (Impotentia coeundi) von Männern gleichermaßen somatische wie psychologische Störungseinflüsse berücksichtigt (s. Tab. 1).

\section{Tab. 1 Ausgewählte andrologische Störungen}

\begin{tabular}{ll} 
Impotentia coeundi & Impotentia generandi \\
erektile Dysfunktion & Subfertilität \\
Mangel an sexuellem Verlangen & Infertilität \\
Ejaculation praecox & \\
Orgasmusstörungen & \\
Störungen der Sexualpräferenz & \\
\hline
\end{tabular}

\subsection{Psychologische Diagnostik bei Fertilitätsproblemen}

\subsubsection{Stress und Subfertilität}

Eine Subfertilität liegt vor, wenn der Patient Spermien produzieren kann, diese jedoch mit hoher Wahrscheinlichkeit für eine natürliche Zeugung nicht ausreichend sind. Hier stellt sich dann die Frage, ob eine eingeschränkte Fertilität möglicherweise auch das Ergebnis von Stressfaktoren sein kann. Aus den dazu vorliegenden Studien konnten bisher folgende Zusammenhänge gefunden werden (Seikowski 2005a):

- negative Korrelationen zwischen sozialer Kompetenz, sozialer Anpassung, Realitätsbezogenheit und Motilität und Morphologie der Spermien

- keine Zusammenhänge zwischen Depressivität und spermiologischen Parametern 
v negative Korrelationen zwischen Hypochondrie und Motilität sowie Morphologie

- Reduktion der Anzahl der Spermien, der Motilität und der Morphologie infolge kritischer Lebensereignisse

v Infertilität als Stressor in Abhängigkeit von Copingstrategien

- Samentransportstörungen

- inadäquates Wissen zum Konzeptionsoptimum

- eingeschränkte Zeugungsfähigkeit in der Paarkonstellation „dominante Frau gefügiger Mann“.

In der Liaisonsprechstunde werden diese prädisponierenden Faktoren mit den Patienten besprochen und es wird ihnen angeboten, mögliche Ursachen mittels einer ausführlichen Anamnese - unterstützt durch testpsychologische Untersuchungen prüfen zu lassen. Sehr oft ergeben sich dann daraus entsprechende individuelle psychologische Interventionsmöglichkeiten.

\subsubsection{Ausbleibender Kinderwunsch bei normalem Spermiogramm}

Auch wenn das Spermiogramm normale Werte zeigt und auch bei der Partnerin keine somatischen Einschränkungen zu finden waren, ist es möglich, dass aufgrund noch anderer Probleme der Kinderwunsch unerfüllt bleibt. Nach unseren Erfahrungen kommen dabei folgende Störungen am häufigsten in Betracht:

- Das Kinderwunschpaar schläft kaum miteinander.

- Der Mann fühlt sich an den fruchtbaren Tagen der Partnerin sehr unter Druck und hat ausgerechnet an diesen Tagen keine vollständige Erektion, sodass das Einführen des Penis in die Vagina nicht möglich ist.

- An den fruchtbaren Tagen der Partnerin bekommt der Mann beim Verkehr keine Ejakulation. Dahinter steckt oft latent die Ablehnung der Zeugung eines Kindes, wodurch eine Ejakulationshemmung die Folge ist.

- Auch ein regelmäßiger vorzeitiger Samenerguss noch vor dem Einführen sollte angesprochen werden.

- Der Mann konnte bisher seine Partnerin befriedigen, kann aber einen Samenerguss nur nach dem Hinausgleiten aus der Vagina im schlaffen Zustand erleben, wodurch auch in diesem Fall eine Zeugung nicht möglich ist (Seikowski 2005b).

- Auch ein Vaginismus der Partnerin sollte hinterfragt werden.

- Wir beobachteten in Einzelfällen auch latent homosexuelle Männer, die ihre Neigung verleugneten und hofften, durch eine heterosexuelle Beziehung sexuell „normal“ leben und eine Familie mit eigenen Kindern gründen zu können. Doch fiel ihnen der sexuelle Akt mit der eigenen Frau mit zunehmendem Alter immer schwerer.

Für all diese Problemkonstellationen sind psychotherapeutische Hilfen vorhanden.

\subsection{Psychologische Diagnostik bei sexuellen Funktionsstörungen}

Wenn Männer mit sexuellen Funktionsstörungen in die Sprechstunde kommen und wesentliche organische Störungen bereits ausgeschlossen werden konnten, muss 


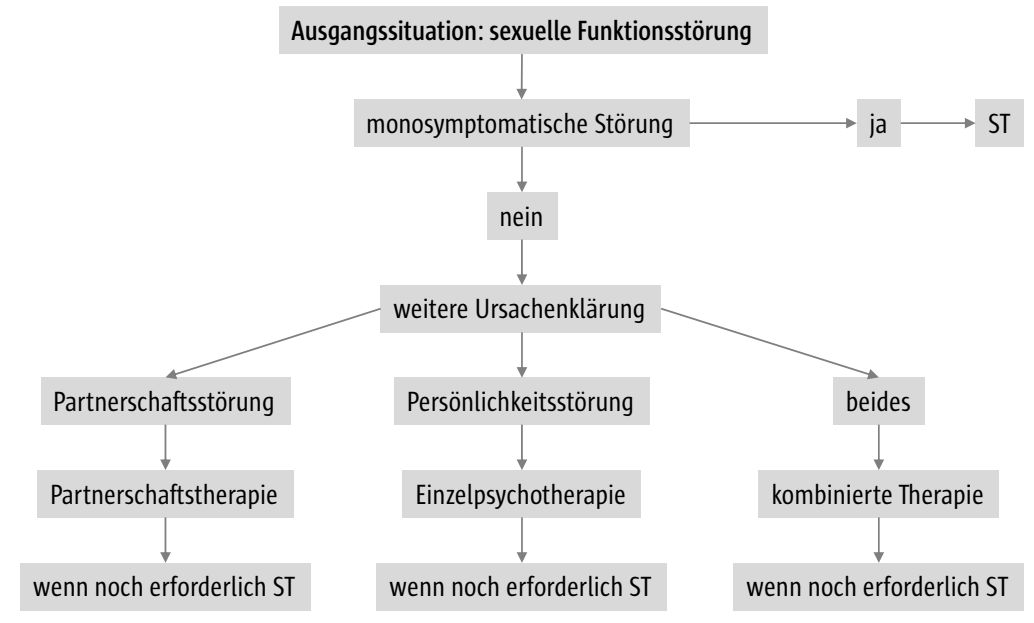

Abb. 1 Psychologische Diagnostik bei sexuellen Funktionsstörungen (ST = Sexualtherapie)

nicht immer gleich mit Medikamenten symptomorientiert behandelt werden. Zunächst sollte festgestellt werden, ob die sexuelle Störung die Hauptstörung darstellt, oder ob sie ein ,kleineres“ Problem einer übergeordneten Störung ist (s. Abb. 1). Partnerschaftsstörungen und/oder Persönlichkeitsstörungen können vorhanden sein. Wenn dem so ist, dann ergeben sich nicht unbedingt sofortige Indikationen für eine spezifische Sexualtherapie (ST), denn dann sollte zunächst die Hauptstörung behandelt werden. Häufig ist dann eine störungsspezifische Sexualtherapie nicht mehr erforderlich.

Stellen die sexuellen Funktionsstörungen jedoch die Hauptstörung dar, dann gibt es eine ganze Reihe besonders verhaltenstherapeutisch orientierter Behandlungsmethoden, die ausführlichst in der Literatur dargestellt sind (vgl. Kockott u. Fahrner 2000; Fahrner u. Kockott 2003).

\subsection{Psychologische Interventionen bei andrologischen Störungen}

\subsubsection{Psychologische Therapieansätze}

Zusätzlich zu den störungsspezifischen Behandlungsmethoden der Sexualtherapie richten sich weitere psychotherapeutische Interventionen jeweils danach, welche Art der psychischen Belastung als vordergründig diagnostiziert wurde:

- Bei den chronischen psychischen Belastungen, die sich in Partnerproblemen, Depressionen und übersteigerten sexuellen Leistungsansprüchen äußern, stehen Partnerschafts- und/oder Einzelpsychotherapien und verschiedene Formen von Sexualberatung und Sexualtherapien im Vordergrund.

- Akute psychische Belastungen spiegeln sich bei Versagensängsten und Formen von Leistungsdruck unmittelbar vor oder beim Verkehr wider - egal ob mit oder ohne Kinderwunsch. Hier sind desensibilisierende Verfahren zu bevorzugen.

- Bei Alltagsbelastungen in Form von Stimmungsschwankungen sowie Entspannungsdefiziten - z.B. bei hoher beruflicher Belastung - werden psychophysio- 
logische Entspannungstechniken bevorzugt (z.B. das Autogene Training und die progressive Muskelrelaxation).

- Bei Dauerbelastungen durch irreversible andrologische Störungen stehen Fragen der Krankheitsbewältigung im Vordergrund.

\subsubsection{Beratung und lösungsfindungen bei irreversiblen andrologischen Störungen}

Egal ob bei sexuellen Funktionsstörungen oder bei Fertilitätsproblemen - das Ziel psychologischer und/oder somatischer Interventionen muss nicht immer die Wiederherstellung der ursprünglichen Sexualität oder die Lösung des Kinderwunsches sein, bei der beide Personen die biologischen Eltern sind. In Tabelle 2 sind die wichtigsten Interventionsvarianten zusammengestellt.

Tab. 2 Beratung und Lösungsfindungen bei irreversiblen andrologischen Störungen

\begin{tabular}{ll} 
Impotentia coeundi & Impotentia generandi \\
\hline nichtvaginale Sexualität & kein „Kind um jeden Preis“ \\
\hline Masturbation als Alternative & Adoption \\
\hline Orientierung auf andere lustbetonte Lebensinhalte & maritogene bzw. donogene Insemination \\
\hline Nachdenken über medikamentöse bzw. technische & Nachdenken über neuere reproduktionsmedizinische \\
Hilfsmittel wie SKAT, Erektionspumpe, MUSE u.a. & $\begin{array}{l}\text { Möglichkeiten zur Kinderwunscherfüllung (ICSI, } \\
\text { MESA, TESE) }\end{array}$ \\
\hline
\end{tabular}

Auf eine Methode soll an dieser Stelle jedoch noch etwas differenzierter bei der Lösung des Kinderwunsches eingegangen werden, weil sie sich hinsichtlich der biologischen bzw. sozialen Stellung von Mann und Frau hinsichtlich der anderen reproduktionsmedizinischen Methoden unterscheidet. Bei der Adoption sind beide Partner nicht die biologischen aber gleichgestellt die sozialen Eltern. Bei den neueren reproduktionsmedizinischen Maßnahmen (ICSI - interzelluläre Spermieninjektion, MESA - mikrochirurgische epididymale Spermienaspiration, TESE - testikuläre Spermienextraktion) sind Mann und Frau in der Regel die biologischen und sozialen Eltern. Dabei wird angenommen, dass es bei einer Gleichstellung beider Geschlechter hinsichtlich des Beziehungsverhältnisses weniger zu psychologischen Problemen kommt als bei der donogenen Insemination (auch als therapeutische donogene Insemination bezeichnet -TDI), bei der die Frau die biologische und die soziale Mutterrolle, der männliche Partner jedoch „nur“ die soziale Vaterrolle übernimmt. Dem Mann wird u.U. sogar die Fähigkeit abgesprochen, mit einer solchen Situation zurechtkommen zu können (Petersen 1987).

Wie in mehreren prospektiven und katamnestischen Untersuchungen zur donogenen Insemination gezeigt werden konnte (Seikowski u. Glander 1996) ist dem jedoch nicht so. Entscheidend für die Akzeptanz dieser Form der Kinderwunscherfüllung sind Partnerschaftsstabilität und das Zusammensein mit dem Kind. D.h. die soziale Vaterrolle ist entscheidender als die biologische. Hinzu kommt, dass die Erfolgsquote bei der donogenen Insemination deutlich höher ist (bis zu über 70\%) als bei den anderen reproduktionsmedizinischen Maßnahmen (Glander et al. 1998). 


\section{Literatur}

Fahrner EM, Kockott G (2003) Sexualtherapie. Ein Manual zur Behandlung sexueller Funktionsstörungen bei Männern. Göttingen: Hogrefe

Glander HJ, Seikowski K, Haake KW (1998) 12 Jahre therapeutische donogene Insemination (TDI) von Kryosperma. Ein Erfahrungsbericht über 3474 Inseminationszyklen. Magyar Andrologia 3, 67-75

Kockott G, Fahrner EM (2000) Sexualstörungen des Mannes. Stuttgart: Thieme

Petersen P (1987) Manipulierte Fruchtbarkeit. Fertilität 3, 99-109

Seikowski K (2005a) Stress and Infertility. Andrologia 37, 109-111

Seikowski K (2005b) Masturbation. In: Schill WB, Bretzel RG, Weidner W (Hrsg.) MännerMedizin in der allgemeinmedizinischen und internistischen Praxis. Urban \& Fischer, 119-121

Seikowski K, Glander HJ (1996) Psychosoziale Aspekte der heterologen Insemination. Der Frauenarzt 37, 117-131 